\title{
Regulatory B cells in experimental mouse models of
}

\section{arthritis}

Diana E. Matei, Claudia Mauri and Elizabeth C. Rosser

Centre for Rheumatology, Division of Medicine, University College London, 5

University Street, London WC1E 6JF, United Kingdom

Correspondence to:

Diana E. Matei

e-mail: diana.matei.16@ucl.ac.uk

Running title: Bregs in experimental arthritis. 


\section{Abstract}

Regulatory B cells (Breg) have been shown to have a role in the suppression of a wide variety of immune responses, yet they are deficient or defective in autoimmune diseases such as rheumatoid arthritis. For the study of autoimmune inflammation, experimental models of arthritis have acted as a valuable tool in understanding the development of Bregs and their role in maintaining immune homeostasis. In this chapter we will focus on the study of transitional-2 marginal zone precursor (T2-MZP) Bregs in the context of two experimental arthritis models: antigen-induced arthritis (AIA) and collagen-induced arthritis (CIA). We will specifically focus on how to induce arthritis, as well as on methods for the isolation and functional study of Bregs both in vitro and in vivo.

Key words: Arthritis, Regulatory B cells, Inflammation, IL-10, Autoimmunity, T2-MZP B cells. 


\section{Introduction}

Our group and others have shown that regulatory B cells (Breg) develop in response to inflammatory cues with the main aim of preventing the excessive damage caused by unrestrained inflammation. Experimental models of arthritis have been used as a model of inflammation to elucidate many intricacies of Breg development and function. For instance, by using the collagen-induced arthritis (CIA) model, previous work by this laboratory has revealed the importance of anti-CD40 stimulation in inducing IL-10 production and supporting the suppressive function of Bregs. CIA has also been pivotal in allowing us to identify splenic transitional-2 marginal zone precursor B cells (T2-MZP) as a candidate cell for a Breg phenotype [1,2]. Moreover, by using the antigen-induced arthritis (AIA) model, we have also identified that the lack of arthritogenic inflammation, observed when mice are treated with gut microbiotadepleting antibiotics, leads to a significant reduction in the number of functional Bregs [3]. The use of the AIA model has also previously revealed that Bregs have the ability to support regulatory $\mathrm{T}$ cell (Treg) differentiation at the expense of pro-inflammatory $\mathrm{CD}^{+}$and $\mathrm{CD}^{+} \mathrm{T}$ cell differentiation [4]. Although the suppressive function of Bregs is now well established, their phenotype has still not been fully elucidated and it has been a hope in the field that a transcription factor specific for Bregs, similar to Foxp3 for Tregs, will eventually be found. Recent work from our group has shown, with the use of the AIA model, that the aryl hydrocarbon receptor (AhR) is highly expressed in IL$10^{+} \mathrm{CD} 19^{+} \mathrm{CD} 21^{\mathrm{hi}} \mathrm{CD} 24^{\mathrm{hi}}$ Bregs, and that this transcription factor skews their differentiation from pro-inflammatory B cells into regulatory B cells, finding supported by the exacerbated arthritis developed by B cell-specific AhR deficient mice [5].

These two models of arthritis are induced models, each with their own advantages and limitations. CIA is the most commonly used model of arthritis and is induced using 
an autoantigen, type II collagen, emulsified in complete Freund's adjuvant (CFA) to break immunological tolerance $[1,2,6,7]$. This leads to the development of a polyarthritis, characterized by a monophasic inflammatory reaction of affected joints, including synovial hyperplasia and cartilage degradation. Though widely used, there are limitations associated with $\mathrm{CIA}$, including problems for the inexperienced researcher setting up the model, as well as the fact that the housing conditions of experimental animals greatly affect the incidence and severity of arthritis. Furthermore, $\mathrm{CIA}$ is only successfully established in certain genetically susceptible mouse strains, such as the DBA/1, as there is a strong link between MHC class II haplotype and disease induction in this model [8].

Conversely, AIA is induced using a two-phase protocol that is based on firstly priming the immune system to methylated bovine serum albumin (mBSA) emulsified with CFA, followed one week later by an intra-articular injection of mBSA, which induces joint inflammation. The resulting mono-arthritic disease is defined by intense synovitis and pannus formation in the injected joint, although it does hold the disadvantage of an acute phase model where joint disease abates from day 3 , therefore not fully representing human rheumatoid arthritis and its chronicity $[4,9,10]$. However, this model holds many advantages for the researcher, including $100 \%$ incidence in the C57BL/6 background which allows for the use of many available transgenic mice, a rapid disease course and, as arthritis is induced in the larger knee joint rather than the smaller joints of the paw, the ability to dissect out the inflamed synovia allowing direct analysis of cellular infiltrates by flow cytometry.

Here, we describe how to efficiently induce CIA and AIA, and how to assess the severity of arthritis. Furthermore, we show how CIA and AIA can be used as models of inflammation to assess the suppressive function of Bregs. Assessment of 
suppression by Bregs remains the gold standard for their identification, due to the heterogeneity of identified Breg phenotypes, such as the $B 10$ cells with $C D 5^{+} C D 1 \mathrm{~d}^{\text {hi }}$ phenotype, the transitional-2 marginal zone precursor (T2-MZP) Bregs with CD $19^{+} \mathrm{CD} 21^{\text {hi }} \mathrm{CD} 24^{\mathrm{hi}} \mathrm{CD} 23^{+}$phenotype, the Tim- $1^{+}$Bregs, and so on [11]. However, as we and others have identified T2-MZPs as B cells with the ability to suppress arthritis and other chronic inflammatory conditions, we will focus on how to assess the suppressive capacity of T2-MZPs both in vivo, using an adoptive transfer technique, and in vitro, using a standard suppression assay approach [1,4,12]. 


\section{Materials}

Prepare all solution in a sterile hood. Prepare and store all reagents at $4^{\circ} \mathrm{C}$ (unless otherwise indicated). All solutions (apart from adjuvants) used for both antigen- and collagen-induced arthritis are endotoxin free.

\subsection{Antigen-induced arthritis}

1. 8-12 week old female or male mice (see Note 1).

2. Methylated Bovine Serum Albumin (mBSA): dissolve $80 \mathrm{mg}$ of mBSA in $2 \mathrm{ml}$ of sterile water by agitating overnight at $4^{\circ} \mathrm{C}$. Store at $4^{\circ} \mathrm{C}$ for up to 6 months.

3. Complete Freund's adjuvant (CFA): in a fume hood, whilst wearing a facemask for safety, finely powder M. tuberculosis with a pestle and mortar. Resuspend powdered bacteria in incomplete Freund's adjuvant (IFA) before transferring into a sterile container. Final concentration should be $3 \mathrm{mg}$ of $M$. tuberculosis per $\mathrm{ml}$ of IFA.

4. Needles: $25 \mathrm{G} \times 5 / 8$ " Needles $(0.5 \times 16 \mathrm{~mm}), 1 \mathrm{ml}$ syringes, $29 \mathrm{G} \times 1 / 2$ " Insulin syringes $(0.33 \times 12 \mathrm{~mm})$.

5. Gaseous Isoflurane: dial vaporizer to OFF and fill with isoflurane. Turn on $\mathrm{O}_{2}$ flow rate to $1 \mathrm{l} / \mathrm{min}$ and set vaporizer to $3 \%$ (see Note 2).

6. Caliper (mechanical external measuring gage).

\subsection{Collagen-induced arthritis}

1. Bovine type II collagen (see Note 3 ).

2. $10 \mathrm{mM}$ acetic acid.

3. 8-12 week old male DBA/1 $\left(\mathrm{H}-2^{\mathrm{q}}\right)$ mice. 
4. Complete Freund's adjuvant: see above Subheading 2.1, step 3 with the following change: final concentration should be $4 \mathrm{mg}$ of $M$. tuberculosis per $\mathrm{ml}$ of IFA.

5. Needles: $25 \mathrm{G} \times 5 / 8 "(0,5 \times 16 \mathrm{~mm})$ needles and $1 \mathrm{ml}$ syringes.

6. Gaseous Isoflurane: see above section 2.1, step 5.

7. Caliper (mechanical external measuring gage).

\subsection{Adoptive transfer of T2-MZPs}

1. Spleens from mice in remission from arthritis (see Note 4).

2. $5 \mathrm{ml}$ syringes.

3. $70 \mu \mathrm{m}$ cell strainers.

4. $15 \mathrm{ml}$ and $50 \mathrm{ml}$ tubes.

5. Polypropylene tubes for cell staining.

6. Red Blood Cell lysis buffer.

7. Recovery medium: $1 \%$ penicillin-streptomycin in RPMI-1640 medium.

8. Supplemented RPMI-1640: 10\% fetal calf serum (FCS), $1 \%$ penicillinstreptomycin, and $50 \mu \mathrm{M}$ 2-mercaptoethanol in RPMI-1640 medium.

9. Magnetic activated cell sorting (MACS) Buffer: 0.5\% FCS, 2 mM ethylenediaminetetraacetic acid (EDTA) in 1X PBS.

10. Kit and associated equipment for immunomagnetic isolation of $B$ cells (see Note 5).

11. Fluorochrome conjugated anti-mouse CD19 (1D3), CD21 (7G6), CD23 (B3B4) and CD24 (M1/69) antibodies.

12.4',6-diamidino-2-phenylindole (DAPI): stock solution $1 \mathrm{mg} / \mathrm{ml}$.

13. Hanks' Balanced Saline solution. 
$14.29 \mathrm{G} \times 1 / 2 "$ Insulin syringes $(0.33 \times 12 \mathrm{~mm})$.

15. Cell sorter.

\subsection{In vitro suppression assay}

1. Spleens from mice in remission from arthritis.

2. $5 \mathrm{ml}$ syringes.

3. $70 \mu \mathrm{m}$ cell strainers.

4. $15 \mathrm{ml}$ and $50 \mathrm{ml}$ tubes.

5. Supplemented RPMI-1640: 10\% FCS, 1\% penicillin-streptomycin, $50 \mu \mathrm{M} 2-$ mercaptoethanol in RPMI-1640 medium.

6. MACS Buffer: 0.5\% FCS, 2 mM EDTA in $1 X$ PBS.

7. Kit and associated equipment for immunomagnetic isolation of B cells.

8. Fluorochrome conjugated anti-mouse CD19 (1D3), CD21 (7G6), CD23 (B3B4), CD24 (M1/69), CD4 (RM4-5) and CD25 (PC61.5) antibodies as well as fluorochrome conjugated mAbs against mouse cytokines of interest (e.g. IL-10, TNF $\alpha$, IL-17, IFN $\gamma)$.

9. Fluorochrome conjugated anti-mouse Foxp3 and anti-mouse Ki67 antibodies. 10.4',6-diamidino-2-phenylindole (DAPI): stock solution $1 \mathrm{mg} / \mathrm{ml}$.

11. Anti-CD3 (145-2C11) antibody, cell culture grade.

12. Phorbol 12-myristate 13-acetate (PMA): dilute in the appropriate medium at the final concentration of $50 \mathrm{ng} / \mathrm{ml}$.

13. Ionomycin: dilute in the appropriate medium at the final concentration of 250 $\mathrm{ng} / \mathrm{ml}$.

14. Brefeldin A: dilute in the appropriate medium at the final concentration of 5 $\mu \mathrm{g} / \mathrm{ml}$. 
15. Fixation and permeabilization buffers for intranuclear staining of transcription factors.

16. Fixation and permeabilization buffers for intracellular staining of cytokines.

17. Fluorescence-activated cell sorting (FACS) buffer: 0.05\% FCS, 2 mM EDTA and $0.001 \%$ sodium azide in $1 \mathrm{X}$ PBS. 


\section{Methods}

Arthritis induction protocols are for 10 mice. Scale up volumes accordingly. All cell suspensions and associated buffers should be kept ice cold for cell isolation and collagen extraction.

\subsection{Induction of antigen-induced arthritis}

1. Add $100 \mu \mathrm{l}$ of the $40 \mathrm{mg} / \mathrm{ml} \mathrm{mBSA}$ stock solution, $900 \mu \mathrm{l}$ of PBS, and $1 \mathrm{ml}$ of CFA in a sterile container. Using a $1 \mathrm{ml}$ syringe, mix solution vigorously until mBSA is fully emulsified in CFA (see Note 6).

2. Place mice in induction chamber and anaesthetize using gaseous isoflurane. Move mice from induction chamber to individual nose cone face-down, and, using clippers, shave the area at the base of the back/pelvis proximal to the tail.

3. Draw up $100 \mu \mathrm{l}$ of $\mathrm{mBSA} / \mathrm{CFA}$ mixture into a $1 \mathrm{ml}$ syringe with a $25 \mathrm{G}$ needle attached, being careful to exclude air bubbles.

4. Inject the full $100 \mu \mathrm{l}$ of $\mathrm{mBSA} / \mathrm{CFA}$ mixture into the subcutaneous fat at the base of the back at one or multiple sites, entering the skin just to the side of the spinal column. Transfer the mouse to a fresh cage to recover from the anesthesia. Immunize remaining animals using a fresh needle per cage or more frequently if needle becomes blunted.

5. Allow mice to rest for seven days checking injection site daily (see Note 7).

6. After seven days, dilute $100 \mu \mathrm{l}$ of the $40 \mathrm{mg} / \mathrm{ml} \mathrm{mBSA}$ stock solution in 100 $\mu \mathrm{l}$ of PBS in a $1.5 \mathrm{ml}$ tube. Mix solution by pipetting up and down. Prepare a $1.5 \mathrm{ml}$ tube containing PBS alone as a control. 
7. Measure size of both knees from each mouse using calipers. Then place mice in induction chamber and anaesthetize using gaseous isoflurane. Move animals from induction chamber to individual nose cone face-up and, using clippers, shave the area around the knee joints.

8. Place $10 \mu \mathrm{l}$ of $\mathrm{mBSA} / \mathrm{PBS}$ mixture into a $29 \mathrm{G}$ insulin syringe and inject under the intra-articular ligament of the left knee. The needle should only be inserted slightly beyond the bevel. If the needle is in the joint, the knee should balloon slightly upon injection of mBSA/PBS. The right knee should receive an intra-articular injection of $10 \mu \mathrm{l}$ of PBS as a control. Transfer mouse to a fresh cage to recover from anesthesia. Immunize remaining animals.

9. On each subsequent day post-intra-articular injection assess disease severity by assessing walking score and knee swelling. The walking score is graded as follows: 4, no walking; 3 , walking on three legs; 2 , limping on the inflamed leg; 1, limping on the inflamed leg only after pressure on the knee; and 0 , normal walking. Knee swelling is measured using calipers and calculated as the percentage increase in knee size as compared with day 0 (pre-injection), or as a percentage difference between mBSA and PBS injected knees (see Note 8).

10. Following remission from arthritis, knee joints can be removed and fixed in formalin $(10 \% \mathrm{v} / \mathrm{v})$ for later analysis by histology.

11. When ready, joints are decalcified in EDTA ( $5 \% \mathrm{w} / \mathrm{v}), \mathrm{pH} 7.3$. Joints are placed in at least 15 volumes of EDTA for 2 weeks at $4^{\circ} \mathrm{C}$ with occasional agitation. Depending on success of decalcification, EDTA solution can be replaced daily. Joints are rinsed, embedded in paraffin, then sectioned and stained with hematoxylin and eosin. The severity of joint inflammation is 
graded as follows: Normal, no damage; Mild, minimal synovitis, cartilage loss and bone erosion limited to discrete foci; Moderate, synovitis and erosion present but normal, intact joint architecture; Severe, synovitis, extensive erosion and joint architecture disrupted.

\subsection{Induction of collagen-induced arthritis}

1. This protocol has been adapted from a previously published protocol [6].

2. Dissolve type II collagen in $10 \mathrm{mM}$ acetic acid according to the manufacturer's instructions overnight at $4^{\circ} \mathrm{C}$, with vigorous mixture, to a final concentration of $4 \mathrm{mg} / \mathrm{ml}$. Dissolved collagen can be stored at $-80^{\circ} \mathrm{C}$ until needed. Solution must be fully defrosted (on ice) before use.

3. Add $0.5 \mathrm{ml}$ of dissolved type II collagen and $0.5 \mathrm{ml}$ of CFA to a sterile container (or adjust volumes according to number of mice in a 1:1 mix). Using a $1 \mathrm{ml}$ syringe, mix solution together vigorously until collagen is fully emulsified in CFA. Emulsion should be thick enough to stay in container when upturned. Keep solution on ice.

4. Place mice in induction chamber and anaesthetize using gaseous isoflurane. Move mice from induction chamber to individual nose cone face-down, and, using clippers, shave the area at the base of the back/pelvis proximal to the tail. Draw up $50 \mu \mathrm{l}$ of collagen/CFA emulsion into a $1 \mathrm{ml}$ syringe with a $25 \mathrm{G}$ needle attached, being careful to exclude air bubbles.

5. Inject emulsion intra-dermally in the skin of the tail (inject at multiple sites if needed, in order to inject the total $50 \mu \mathrm{l})$. Immunize remaining animals using a fresh needle per cage or as needle becomes blunted. 
6. Check mice for arthritis development every day two weeks postimmunization (see Note 9). Visible clinical arthritis usually develops around day 30 .

7. Following arthritis onset, the clinical severity of arthritis is determined by measuring swelling of affected paws with calipers or by using the following scoring system. Arthritis is graded as follows: 3 , ankylosis; 2, pronounced swelling; 1 , slight swelling and/or erythema; 0, normal. Each limb is graded individually, thus there is a maximum score of 12 per mouse. The percentage incidence of arthritis amongst the experimental cohort can also be recorded.

8. Following remission from arthritis, affected paws can be removed and fixed in formalin $(10 \% \mathrm{v} / \mathrm{v})$ for later analysis by histology.

9. When ready, joints are then prepared and assessed for damage to articular structures (see Subheading 3.1, step 11). Alternatively, the proportion of eroded joints (defined as cartilage or bone with demarcated defects or filled with inflammatory tissue) can be determined.

\subsection{Adoptive transfer of T2-MZPs (in vivo suppression assay)}

As mentioned above, due to the heterogeneity of the known Breg phenotypes, the assessment of their suppressive function is the gold standard in their identification. Here we describe how to determine the suppressive capacity of transitional-2 marginal zone precursor (T2-MZP) Bregs with a CD19 ${ }^{+} \mathrm{CD} 21^{\text {hi }} \mathrm{CD} 24^{\text {hi }} \mathrm{CD} 23^{+}$phenotype in vivo. The T2-MZP Breg population is expanded and activated in mice in remission from arthritis, which is why we use this particular time point in disease to isolate them. 


\subsubsection{Purification of T2-MZP B cells}

1. Dissect spleens from mice in remission from arthritis, put in tubes containing ice cold recovery medium, and place directly on ice (see Note 10).

2. In a sterile hood, mash spleens through a $70 \mu \mathrm{m}$ cell strainer (maximum 3 per strainer) into a $50 \mathrm{ml}$ tube using the flat end of the plunger from a $5 \mathrm{ml}$ syringe. Wash through filter with $15 \mathrm{ml}$ of supplemented RPMI. Centrifuge for $10 \mathrm{~min}, 4^{\circ} \mathrm{C}, 500 \times \mathrm{g}$.

3. Discard supernatant and resuspend in $1 \mathrm{ml} / \mathrm{spleen}$ of red cell lysis buffer, leave for 2 min (see Note 11). Following incubation with red lysis buffer, fill tube to the top with supplemented RPMI. Centrifuge for $10 \mathrm{~min}, 4^{\circ} \mathrm{C}, 500 \mathrm{x}$ g.

4. Resuspend cells in $10 \mathrm{ml}$ of MACS buffer per spleen and count. Centrifuge for $10 \mathrm{~min}, 4^{\circ} \mathrm{C}, 500 \times g$.

5. Negatively isolate resting B cells using immunomagnetic separation technology. Follow the instructions of the employed kit and, at the end, count isolated cells. Centrifuge for $10 \mathrm{~min}, 4^{\circ} \mathrm{C}, 500 \times \mathrm{g}$.

6. Resuspend cells at $50 \times 10^{6}$ per $\mathrm{ml}$ in $1 \mathrm{X}$ PBS and stain with suggested antibody clones against CD19, CD21, CD23 and CD24 at a dilution of 1 in 125. Other clones will have to be optimized. Concurrently, prepare single stain controls for the compensation of the flow cytometer. Stain on ice for 20 $\min$.

7. In the meantime, prepare collection tubes using polypropylene FACS tubes. Add 1-2 $\mathrm{ml}$ of a 1:1 mixture of FACS and MACS buffer to each $5 \mathrm{ml}$ collection tube. 
8. Following staining, wash cells twice in MACS buffer and resuspend at $30-50$ x $10^{6}$ cells per $\mathrm{ml}$ or appropriate concentration for cell sorter.

9. Transfer sample into filter-topped polypropylene FACS tubes, filtering through the lids to remove clumps in order to prevent blockage in the cell sorter.

10. Add DAPI at a final concentration between 0.05 and $0.1 \mu \mathrm{g} / \mathrm{ml}$ to the sample and to the single stained control. The staining with this fluorescent dye allows to differentiate between live and dead cells during sorting.

11. Take sample to cell sorter and sort T2-MZPs (see Fig. 1). Begin by excluding cellular debris and doublets using the forward and side scatter gating, followed by dead-cell exclusion using DAPI, and then selecting CD19+ B cells. Within the B cell gate, CD21 and CD24 expression allows exclusion of follicular B cells. Within the CD2 ${ }^{\text {hi }} C D 24^{\text {hi }} \mathrm{T} 2$ and $\mathrm{MZ}$ (marginal zone B cells) gate, CD23 will allow for the final sorting of T2-MZP Bregs, as MZ B cells do not express this marker. 
A
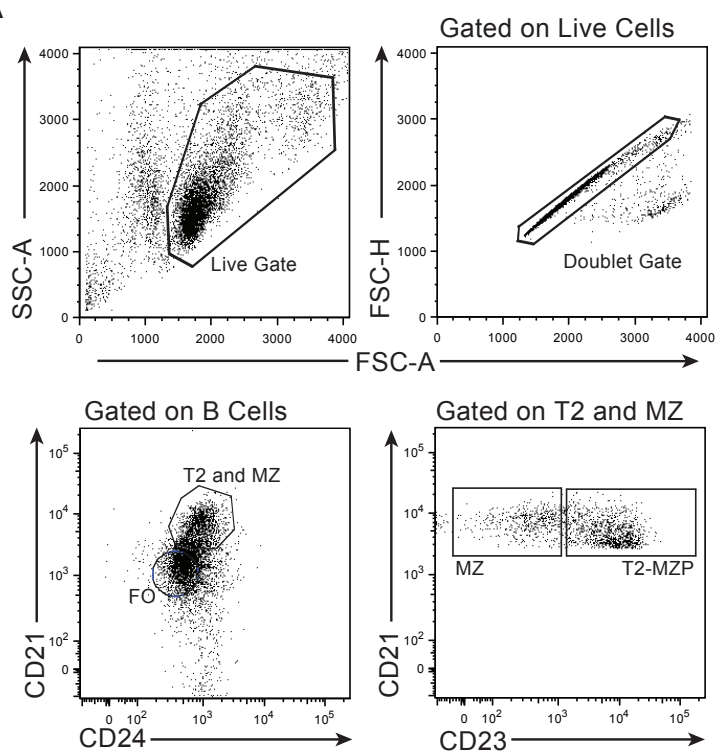

B

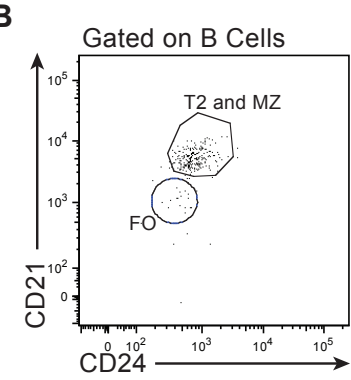

Gated on Doublets

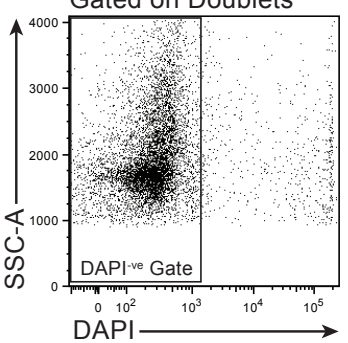

Gated on DAPI-ve

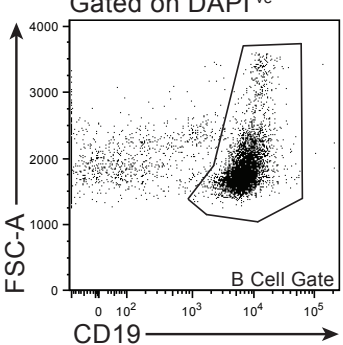

Figure 1: Gating strategy and purity plots for T2-MZP isolation. Isolated B cells from mice in remission from arthritis were stained with antibodies against CD19, CD21, CD23, and CD24 for FACS. (A) Representative flow cytometry plots showing gating strategy for purification of T2-MZP B cells. (B) Representative flow cytometry plots showing purity of isolated T2-MZP B cells post-sort. 


\subsubsection{Adoptive transfer of T2-MZP B cells}

1. Whilst T2-MZPs are being sorted, immunize recipient mice with the intraarticular injection for AIA induction, or with the CII/CFA injection for CIA (see Subheadings 3.1 and 3.2).

2. After samples have been sorted wash cells twice in Hanks' saline solution and count cells (see Note 12).

3. Resuspend T2-MZPs in Hanks' saline solution at appropriate concentration (see Note 13).

4. Heat recipient mice for $15 \mathrm{~min}$ in a heating chamber and inject T2-MZPs intravenously into a tail vein using an insulin needle (use PBS injection as control). Be careful to not aspirate the cells multiple times, as this will damage cellular membranes and possibly lead to cell lysis.

5. Assess arthritis daily on subsequent days.

\subsection{In vitro suppression assay}

The suppressive capacity of T2-MZP Bregs with a CD19+CD21 ${ }^{\text {hi }} \mathrm{CD} 24^{\text {hi }}{ }^{\text {CD } 23^{+}}$ phenotype can also be assessed in vitro, by evaluating their ability to suppress $\mathrm{CD} 4^{+} \mathrm{CD} 25^{-} \mathrm{T}$ cell proliferation, as well as to induce $\mathrm{CD} 4^{+} \mathrm{CD} 25^{+} \mathrm{Foxp} 3^{+}$regulatory $\mathrm{T}$ cells differentiation. The suppressive effect of T2-MZP Bregs does not only reduce $\mathrm{CD}^{+}{ }^{+} \mathrm{CD} 25^{-} \mathrm{T}$ cell proliferation, it also leads to a decrease in the production of proinflammatory cytokines such as IL-17, IFN $\gamma$ and TNF $\alpha$, in favor of the induction of IL10 producing Tregs.

1. Dilute the anti-CD3 antibody to a working concentration of $1 \mu \mathrm{g} / \mathrm{ml}$ in PBS. Add $200 \mu$ l of solution per well of a 96 well-plate and place in incubator. Some wells should be left uncoated for unstimulated controls. 
2. Purify T2-MZP B cells (see Subheading 3.3.1), keeping some splenocytes aside for isolation of $\mathrm{CD}^{+}{ }^{+} \mathrm{CD} 25^{-} \mathrm{T}$ cells.

3. Prepare splenocytes (see Subheading 3.3.1, steps 1-4) and stain with antimouse CD4 and anti-mouse CD25 antibodies at a dilution of 1 in 125 in FACS buffer. Incubate for 20 min on ice and then wash cells twice in MACS buffer. Resuspend cells at the appropriate concentration for cell sorting and sort CD4 ${ }^{+} \mathrm{CD} 25^{-} \mathrm{T}$ cells.

4. After $2 \mathrm{~h}$, aspirate the media from the 96 well plate since the anti-CD3 antibody should be plate bound.

5. Count sorted cells and resuspend in supplemented RPMI. Add T2-MZPs and T cells at a concentration of $1: 1$ into the well (working range is $0.2-0.5$ million cells each). To correctly assess T2-MZP-mediated suppression, include an unstimulated T cell alone control, and an anti-CD3 stimulated T cell alone control. We suggest a minimum of duplicates. Once the cells have been seeded, put the plate in the incubator for $48 \mathrm{~h}$.

6. To determine the suppression of effector T cell proliferation by T2-MZP B cells, collect cells and stain for Ki67 expression. Firstly, wash and stain samples with appropriate cell surface markers to allow for B cell and $\mathrm{T}$ cell differentiation, as per manufacturer's instructions or as described above in the pre-sort staining steps. Wash cells after surface staining and fix and permeabilize the cells with an appropriate intranuclear fixation and permeabilization buffer set, according to kit instructions. Wash cells using an appropriate permeabilization wash buffer, add the anti-Ki67 antibody and incubate for $30 \mathrm{~min}$ at room temperature in the dark. Wash the cells in the same permeabilization buffer and finally resuspend in FACS buffer (see Note 
14). A similar protocol can be used to analyze the expression of intranuclear Foxp3, in order to determine expansion of Tregs following contact with Bregs.

7. Lastly, to determine the effect of Breg suppression on the function of T cells, during the last $5 \mathrm{~h}$ of the $48 \mathrm{~h}$ co-culture, cells can be stimulated with 50 $\mathrm{ng} / \mathrm{ml}$ PMA and $250 \mathrm{ng} / \mathrm{ml}$ ionomycin in the presence of $5 \mu \mathrm{g} / \mathrm{ml}$ Brefeldin A, and then stained intracellularly for the expression of cytokines such as IL-17, IFN $\gamma$, TNF $\alpha$ and IL-10 (see Note 15).

\section{Notes}

1. Incidence of AIA is $100 \%$ regardless of mouse strain and sex. However, severity of disease does vary between strains. C57BL/6 and BALB/C mice are susceptible whereas CBA mice are thought to be relatively resistant.

2. The anesthetic protocol used will depend on the equipment provided by the animal facility. However, we recommend the use of isoflurane as it is less toxic for animals than alternatives and has a faster recovery time. Alternatives include gaseous halothane, or a mixture of xylazine-HCL (5-10 mg/Kg)/ketamine-HCL (50-100 $\mathrm{mg} / \mathrm{Kg}$ ) can be used as an injectable anesthetic. Subcutaneous injections can be performed on un-anesthetized mice, however, for intra-articular injections mice must be fully asleep.

3. We strongly recommend using bovine type II collagen from Chondrex, as we have no experience using other suppliers.

4. The appropriate number of spleens must be calculated depending on strain and type of arthritis. Naïve spleens, or B cells from other organs, can be used, however the protocol must be adjusted accordingly. 
5. As the purposes of this step is only to enrich for B cells in order to make the cell sort run faster than if B cells were sorted directly from splenocytes, several different B cell enrichment kits can be used and, as $100 \%$ purity is not necessary, reagents can be diluted further than recommended. However, we would recommend using kits that negatively select for $B$ cells.

6. Thorough emulsification is required for proper induction of arthritis, we recommend a minimum of 100 strokes with syringe, vortexing alone is not sufficient.

7. A rare side effect of injecting mice with CFA is the formation of an ulcer around the injection site (this is more likely to happen in genetically modified mice such as Rag2 $^{--}$or $\mu \mathrm{MT}$ animals). If this occurs, mice must be checked daily to make sure the wound is dry and healing properly. If not, mice must be sacrificed for humane reasons.

8. In order to keep consistent results, we suggest that the same researcher holds the calipers for every measurement, and that a colleague is employed to hold mice while measurement is taken thus allowing proper extension of the knee joint. Comparison of arthritic knee swelling to swelling of a PBS control injected knee adjusts disease scores to account for injection site inflammation so that only antigen-specific swelling is measured.

9. We usually find it is not necessary, but some researchers find that a booster injection is necessary to induce CIA. Booster injections are carried out by emulsifying collagen 1:1 in incomplete Freund's adjuvant, and $100 \mu$ is injected into mice on day 21 following the first injection.

10. In order to detect T cell suppression, we recommend using donor animals which have the same type of experimental arthritis as recipient mice. The number of 
spleens dissected will depend on the following considerations: we calculate that approximately $0.8 \times 10^{6} \mathrm{~T} 2-\mathrm{MZPs}$ can be isolated from one C57BL/6 mouse in remission from AIA and $1.2 \times 10^{6}$ T2-MZPs can be isolated from one DBA mouse in remission from CIA. Furthermore, in order to see significant suppression in AIA you need to transfer between 1.5-2 x 106 T2-MZPs per mouse, whereas, in CIA you only need to transfer between $4-10 \times 10^{5}$ T2-MZPs per mouse. Thus, the number of spleens dissected must be adjusted depending on the strain, type of arthritis, and number of recipients.

11. We do not recommend lysing more than five spleens together; if more spleens than this are lysed together, we find that cell viability is affected.

12. Do not forget to evaluate cell purities before processing isolated T2-MZPs further. Moreover, if surplus cells have been isolated, we recommend culturing cells with antigen (mBSA or collagen) and/or anti-CD40 (both $10 \mu \mathrm{g} / \mathrm{ml}$ ) for $72 \mathrm{~h}$ to assess IL-10 production by ELISA. IL-10 data can be successfully paired with suppression assay data. Cells can also be put in Trizol ${ }^{\mathrm{TM}}$, or another appropriate extraction buffer, for assessment of gene transcription by RT-PCR.

13. When injecting intended numbers of cells in $100 \mu \mathrm{l}$ volumes, we recommend drawing up $120 \mu \mathrm{l}$ into the syringe to avoid losing cells in the dead space of the needle and syringe barrel (roughly equivalent to $20 \mu \mathrm{l}$ ).

14. Ki-67 antibodies from different sources might suggest specific staining protocols, please follow instructions if different to our description. It is also advisable to titrate antibodies according to manufacturer's instructions in order to determine their working concentration, especially for transcription factor-specific antibodies.

15. We also recommend taking supernatants for assessment of cytokine levels by ELISA. 


\section{Acknowledgements}

This work is funded by a Versus Arthritis program grant (21140) awarded to Claudia

Mauri. Diana Matei is funded by a Versus Arthritis PhD studentship (21257) to C.M.

Elizabeth Rosser is funded by a Versus Arthritis UK Foundation fellowship (21141). 


\section{References}

1. Evans JG, Chavez-Rueda KA, Eddaoudi A, Meyer-Bahlburg A, Rawlings DJ, Ehrenstein MR, Mauri C (2007) Novel suppressive function of transitional 2 B cells in

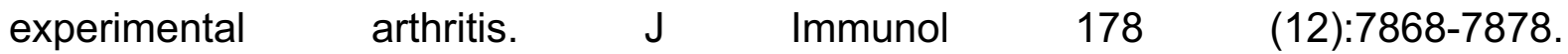
doi:10.4049/jimmunol.178.12.7868

2. Mauri C, Gray D, Mushtaq N, Londei M (2003) Prevention of arthritis by interleukin 10-producing B cells. J Exp Med 197 (4):489-501. doi:10.1084/jem.20021293

3. Rosser EC, Oleinika K, Tonon S, Doyle R, Bosma A, Carter NA, Harris KA, Jones SA, Klein N, Mauri C (2014) Regulatory B cells are induced by gut microbiota-driven interleukin-1beta and interleukin-6 production. Nat Med 20 (11):1334-1339. doi:10.1038/nm.3680

4. Carter NA, Vasconcellos R, Rosser EC, Tulone C, Munoz-Suano A, Kamanaka M, Ehrenstein MR, Flavell RA, Mauri C (2011) Mice lacking endogenous IL-10-producing regulatory $B$ cells develop exacerbated disease and present with an increased frequency of Th1/Th17 but a decrease in regulatory T cells. J Immunol 186 (10):55695579. doi:10.4049/jimmunol.1100284

5. Piper CJM, Rosser EC, Oleinika K, Nistala K, Krausgruber T, Rendeiro AF, Banos A, Drozdov I, Villa M, Thomson S, Xanthou G, Bock C, Stockinger B, Mauri C (2019) Aryl Hydrocarbon Receptor Contributes to the Transcriptional Program of IL-10Producing Regulatory B Cells. Cell Rep 29 (7):1878-1892 e1877. doi:10.1016/j.celrep.2019.10.018

6. Brand DD, Latham KA, Rosloniec EF (2007) Collagen-induced arthritis. Nat Protoc 2 (5):1269-1275. doi:10.1038/nprot.2007.173 
7. Carter NA, Rosser EC, Mauri C (2012) Interleukin-10 produced by B cells is crucial for the suppression of Th17/Th1 responses, induction of T regulatory type 1 cells and reduction of collagen-induced arthritis. Arthritis Res Ther 14 (1):R32. doi:10.1186/ar3736

8. Grotsch B, Bozec A, Schett G (2019) In Vivo Models of Rheumatoid Arthritis. Methods Mol Biol 1914:269-280. doi:10.1007/978-1-4939-8997-3_14

9. Brackertz D, Mitchell GF, Mackay IR (1977) Antigen-induced arthritis in mice. I. Induction of arthritis in various strains of mice. Arthritis Rheum 20 (3):841-850. doi:10.1002/art.1780200314

10. Vasconcellos R, Carter NA, Rosser EC, Mauri C (2011) IL-12p35 subunit contributes to autoimmunity by limiting IL-27-driven regulatory responses. J Immunol 187 (6):3402-3412. doi:10.4049/jimmunol.1100224

11. Rosser EC, Mauri C (2015) Regulatory B cells: origin, phenotype, and function. Immunity 42 (4):607-612. doi:10.1016/j.immuni.2015.04.005

12. Blair PA, Chavez-Rueda KA, Evans JG, Shlomchik MJ, Eddaoudi A, Isenberg DA, Ehrenstein MR, Mauri C (2009) Selective targeting of B cells with agonistic anti-CD40 is an efficacious strategy for the generation of induced regulatory T2-like B cells and for the suppression of lupus in MRL/lpr mice. J Immunol 182 (6):3492-3502. doi:10.4049/jimmunol.0803052 